\title{
Asymmetric microarray data produces gene lists highly predictive of research literature on multiple cancer types
}

\author{
Noor B Dawany, Aydin Tozeren
}

\begin{abstract}
Background: Much of the public access cancer microarray data is asymmetric, belonging to datasets containing no samples from normal tissue. Asymmetric data cannot be used in standard meta-analysis approaches (such as the inverse variance method) to obtain large sample sizes for statistical power enrichment. Noting that plenty of normal tissue microarray samples exist in studies not involving cancer, we investigated the viability and accuracy of an integrated microarray analysis approach based on significance analysis of microarrays (merged SAM) using a collection of data from separate diseased and normal samples.

Results: We focused on five solid cancer types (colon, kidney, liver, lung, and pancreas), where available microarray data allowed us to compare meta-analysis and integrated approaches. Our results from the merged SAM significantly overlapped gene lists from the validated inverse-variance method. Both meta-analysis and merged SAM approaches successfully captured the aberrances in the cell cycle that commonly occur in the different cancer types. However, the integrated SAM analysis replicated the known cancer literature (excluding microarray studies) with much more accuracy than the meta-analysis.

Conclusion: The merged SAM test is a powerful, robust approach for combining data from similar platforms and for analyzing asymmetric datasets, including those with only normal or only cancer samples that cannot be utilized by meta-analysis methods. The integrated SAM approach can also be used in comparing global gene expression between various subtypes of cancer arising from the same tissue.
\end{abstract}

\section{Background}

Microarray studies typically provide intensity levels for thousands of genes. However, not only are the individual datasets usually small in size, but the inferences made from individual studies are often inconsistent with similar studies [1]. As thousands of microarray samples have accumulated in publicly accessible databases in the last decade [2-4], several statistical methods have been developed to allow for the combination and comparison of data from multiple sources. Among the many methodologies that exist, which deal with combining different microarray datasets, are the permutation tests $[5,6]$, parametric tests and clustering [7], rank-aggregation procedures [8,9], rank products [10], METRADISC [1], and inverse-variance [11-13]. The utilization of vast

\footnotetext{
* Correspondence: aydin.tozeren@drexel.edu

Center for Integrated Bioinformatics, Drexel University, Bossone Research Building 711, 3102 Market Street, Philadelphia, PA 19104, USA
}

amounts of microarray data provided by different groups is considered to increase the reliability of the results and weakens the effects of lab-specific noise [14].

The meta-analysis procedures cited above combine results from different studies. Each dataset is analyzed separately. Genes are associated with an effect size or a $\mathrm{p}$-value. These are then combined across all analyses and a top-ranked gene list is generated based on the aggregated effect size or p-value [15]. While some metaanalysis methods require the use of raw data $[5,6,11]$, others can depend solely on the ranking of genes from various studies $[8,9]$. The meta-analysis is robust in the sense that it allows for comparisons across different platforms and analytical techniques (cDNA and oligonucleotide microarrays). However, the most important limitation the meta-analysis poses is that it requires datasets to include both control and test samples. Previous studies showed that aggregating data prior to
C Biomed Central

() 2010 Dawany and Tozeren; licensee BioMed Central Ltd. This is an Open Access article distributed under the terms of the Creative Commons Attribution License (http://creativecommons.org/licenses/by/2.0), which permits unrestricted use, distribution, and reproduction in any medium, provided the original work is properly cited. 
obtaining results is usually more powerful than obtaining separate statistics from each dataset and then integrating the results [16]. Therefore, based on the grounds of previous studies that revealed the predictive potential of integrated microarray [17-19], we consider in this study a large-scale merge approach to the significance analysis of microarrays (SAM; [20]) test that can utilize asymmetric datasets. SAM was chosen as the significance test because it is extensively used in our lab and has previously been used in normal, tumor and cell line comparisons [21]. Its performance has been shown to be superior to that of other conventional microarray analysis methods. Moreover, SAM uses random iterations to calculate the false discovery rate, allowing the user to control and adjust results accordingly [20].

To test the performances of the meta-analysis and the merged SAM approach, we compiled microarray data from 31 laboratories, resulting in a database containing 339 healthy tissue samples and 1,429 cancer samples from 5 different tissue types using comparable Affymetrix platforms. The tumor tissue types considered in this study -colon, kidney, liver, lung, and pancreas - had multiple microarray datasets containing both normal and disease samples. The meta-analysis approach has already been employed by a few cancer microarray studies either focusing on a single tissue type [5,13,22-24] or across different tissues in order to identify gene sets associated with common cancer mechanisms $[6,11,25]$. For the purpose of this study, the inverse-variance (IV) test was adopted from the work of Ramasamy et al. [11] to compare the quality of our results, since it is believed to be the most comprehensive meta-analysis method for two-class microarray gene expression analyses. With this large-scale database we generated significantly altered gene lists for each individual tissue as well as across all five tissue types, using both the IV and the merged SAM tests. Our results show that the merged SAM analysis, when based on large-scale data, not only significantly overlaps the results produced by the IV metaanalysis, but also provides gene lists that replicate the known cancer literature at least as well as the IV test.

\section{Results}

\section{Datasets and approaches}

Three different groups of microarray datasets were used to evaluate (a) the intersection of significant gene lists predicted by meta-analysis and merged SAM methods and (b) compare these predictions with research literature excluding microarray studies. Group 1 is composed of Affymetrix microarray datasets containing both cancer and normal samples for five different cancer tissues (Table 1). The gene set predictions resulting from analysis of this data with the use of meta-analysis and merged SAM are denoted as IV1 and SAM1, respectively. Each
Table 1 Overview of datasets used and distribution of microarray samples

\begin{tabular}{|c|c|c|c|c|c|}
\hline Analysis & Tissue & $\begin{array}{l}\text { Accession } \\
\#\end{array}$ & Normal & Cancer & Platform \\
\hline \multirow{20}{*}{$\begin{array}{l}\text { IV1/IV2/SAM1/ } \\
\text { SAM2 }\end{array}$} & Colon & E-MTAB-57 & 22 & 25 & A \\
\hline & & GSE4107 & 10 & 12 & P2 \\
\hline & & GSE4183 & 8 & 15 & P2 \\
\hline & Kidney & E-TABM-282 & 11 & 16 & P2 \\
\hline & & GSE11024† & 12 & 60 & P2 \\
\hline & & GSE11151 & 3 & 57 & P2 \\
\hline & & GSE14762† & 12 & 10 & P2 \\
\hline & & GSE15641 & 23 & 57 & A \\
\hline & & GSE6344 & 10 & 10 & A \\
\hline & & GSE7023 & 12 & 35 & P2 \\
\hline & Liver & GSE14323 & 19 & 47 & A/A2 \\
\hline & & GSE6764 & 10 & 35 & P2 \\
\hline & Lung & E-MEXP-231 & 9 & 49 & A \\
\hline & & GSE10072 & 49 & 58 & A \\
\hline & & GSE7670 & 27 & 27 & A \\
\hline & Pancreas & $\begin{array}{l}\text { E-MEXP- } \\
1121 \dagger\end{array}$ & 6 & 17 & A \\
\hline & & E-MEXP-950 & 11 & 14 & A \\
\hline & & GSE15471 & 39 & 39 & P2 \\
\hline & & GSE16515 & 15 & 36 & P2 \\
\hline & & Total: & 294 & 619 & \\
\hline \multirow[t]{13}{*}{ SAM2 } & Colon & $\begin{array}{l}\text { E-MEXP- } \\
1224\end{array}$ & 0 & 55 & A \\
\hline & & E-MEXP-383 & 0 & 36 & A \\
\hline & & E-TABM-176 & 55 & 0 & P2 \\
\hline & & GSE12945 & 0 & 36 & A \\
\hline & & GSE17538 & 0 & 232 & P2 \\
\hline & Kidney & GSE10320 & 0 & 144 & A \\
\hline & & GSE11904 & 0 & 21 & $\mathrm{~A} 2$ \\
\hline & Liver & E-TABM-292 & 0 & 32 & A \\
\hline & & E-TABM-36 & 0 & 57 & A \\
\hline & & GSE9843 & 0 & 69 & P2 \\
\hline & Lung & GSE10445 & 0 & 72 & P2 \\
\hline & & GSE12667 & 0 & 75 & P2 \\
\hline & & Total: & 55 & 829 & \\
\hline \multirow[t]{6}{*}{ IV2 } & Colon & GSE6988 & 28 & 52 & cDNA \\
\hline & Kidney & GSE3 & 81 & 90 & CDNA \\
\hline & Lung & GSE7367 & 24 & 24 & cDNA \\
\hline & & GSE2088 & 30 & 57 & cDNA \\
\hline & & GSE8596 & 6 & 69 & cDNA \\
\hline & & Total: & 169 & 292 & \\
\hline
\end{tabular}

† Datasets included replicated samples

Platforms: A: HG-U133A, A2: HG-U133A2, P2: HG-U133 Plus 2

dataset was analyzed separately for the IV1 test and a final gene list was produced based on the weighted results from the individual datasets. The SAM1 test was applied to the same Affymetrix data from each tissue after their merger, with all samples being normalized 
together, regardless of dataset. Group 2 of microarray datasets used in intersection analysis and literature comparison contained cDNA microarray datasets in addition to the Affymetrix data in Group 1 . The gene lists predicted by meta-analysis using these datasets were called IV2. We used Group 2 to take full advantage of the capability of meta-analysis in integrating microarray datasets from different technologies. Group 3 contained asymmetric Affymetrix data in addition to data in Group 1 (Table 1). The gene list corresponding to Group 3 data predicted by merged SAM is referred to as SAM2. Figure 1a shows the overall characteristics of the Affymetrix datasets used in the analysis. The intersections of the predicted gene lists obtained with the two methods on the three different groups of datasets are summarized in Table 2. The table also presents the p-values corresponding to the intersections based on hypergeometric test.

Moreover, to assess the effect of the refRMA method in normalizing data, three samples from different colon datasets (E-MTAB-57, GSE4107 and GSE4183) were chosen. The expression values for the three arrays were obtained based on classical RMA and refRMA normalization techniques. Quantile-quantile plots were produced to compare the distributions of the different datasets in a pair-wise manner (Figure 2). The points within the plot should form a straight line if the two arrays have similar distributions. The results in Figure 2 draw attention to the differences in distributions when normalizing datasets individually using RMA as opposed to refRMA's ability to normalize the different datasets to possess similar distributions.

\section{IV meta-analysis and merged SAM overlap significantly in results}

As in previous microarray studies of cancer [21,26-31], the gene lists produced by the two approaches used in this study indicate significant alterations of the transcriptional profile as the tissue is transformed from the normal to the cancer state, with up to thousands of genes possibly undergoing statistically significant expression changes. While the two methods applied to the three dataset groups produced different lists of significant genes for each of the five tissues under consideration, there was a considerable overlap in the results (Table 2). The significance of the intersection between predicted gene lists increased consistently as the number of top-ranked genes used in comparison were increased from 10 to 400 . In colon tissue, the overlap with IV1 was confined to 338 significant genes instead of 400 , since that was the total number of genes passing the test criteria. At the 400 gene level p-values of the IV1/ SAM1 intersection ranged from $2.66 \mathrm{E}-26$ in pancreas to 8.42E-181 in lung, while the most significant overlap in
IV1/SAM2 was in kidney (p-value $=1.02 \mathrm{E}-134)$. Comparison of the results of the two SAM methods produced even larger commonalities in the gene lists identified. Apart from the colon tissue, there was at least $60 \%$ overlap between the top 400 gene-lists generated by the two SAM methods, for any given comparison. The match between the two SAM results became less pronounced with sharp increases in the number of samples added in SAM2. Nevertheless, even with 506 colon cancer samples included in SAM2 as opposed to the 92 used in SAM1, the overlap between the two methods (176 genes) remained significant. The overlap between IV1 and IV2 varied largely among the top ranked 400 genes with a minimum overlap of 144 genes in lung tissue and a maximum overlap of 355 genes in kidney, resulting in vanishing $\mathrm{p}$-values in the latter case (Table 2).

To identify significantly altered genes across the five considered tissue types, the datasets from all tissues were pooled together. Again, SAM2 included additional datasets with cancer or normal samples only. Similarly, the significance of the overlap between the results increased as more top-ranked genes were considered, with p-values equal to $6.82 \mathrm{E}-97$ and $2.80 \mathrm{E}-103$ for the intersection at the top 400 genes level in IV1/SAM1 and IV1/SAM2, respectively (Table 2).

\section{Cell cycle pathway and mitosis-related cell division biological processes are commonly enriched in cancers}

The cellular pathways and biological processes that were statistically enriched in the top 400 cancer-associated genes from the multiple tissues under consideration were identified using the DAVID Bioinformatics Resources' [32,33] functional annotation tool as described in the Methods section. Enriched KEGG [34] pathways common to at least 2 tissue types within a given test method or significantly associated with the combined 5-tissue comparisons are shown in Figure 3. The cell cycle pathway was statistically enriched in IV1, IV2, SAM1 and SAM2 gene lists across all tissue types (Figure 4). Among the key changes in the cell cycle in normal to cancer transition are the differential expression of cyclins (A and B) and cyclin-dependent kinases (CDK1 and CDK4/6 complex). CDKs are the core of the regulatory apparatus of the cell cycle progression as changes in the kinases and cyclins drive the cell from one stage of the cell cycle to another [35].

In addition, the p53 signaling pathway and purine metabolism were significantly enriched in all-tissue analyses of both IV tests and SAM2. Pyrimidine metabolism is also enriched for the merged SAM2 significant genes while SAM1 genes are associated with ECM-receptor interaction and glycolysis/gluconeogenesis pathways. At the tissue level, some of the metabolic pathways were 


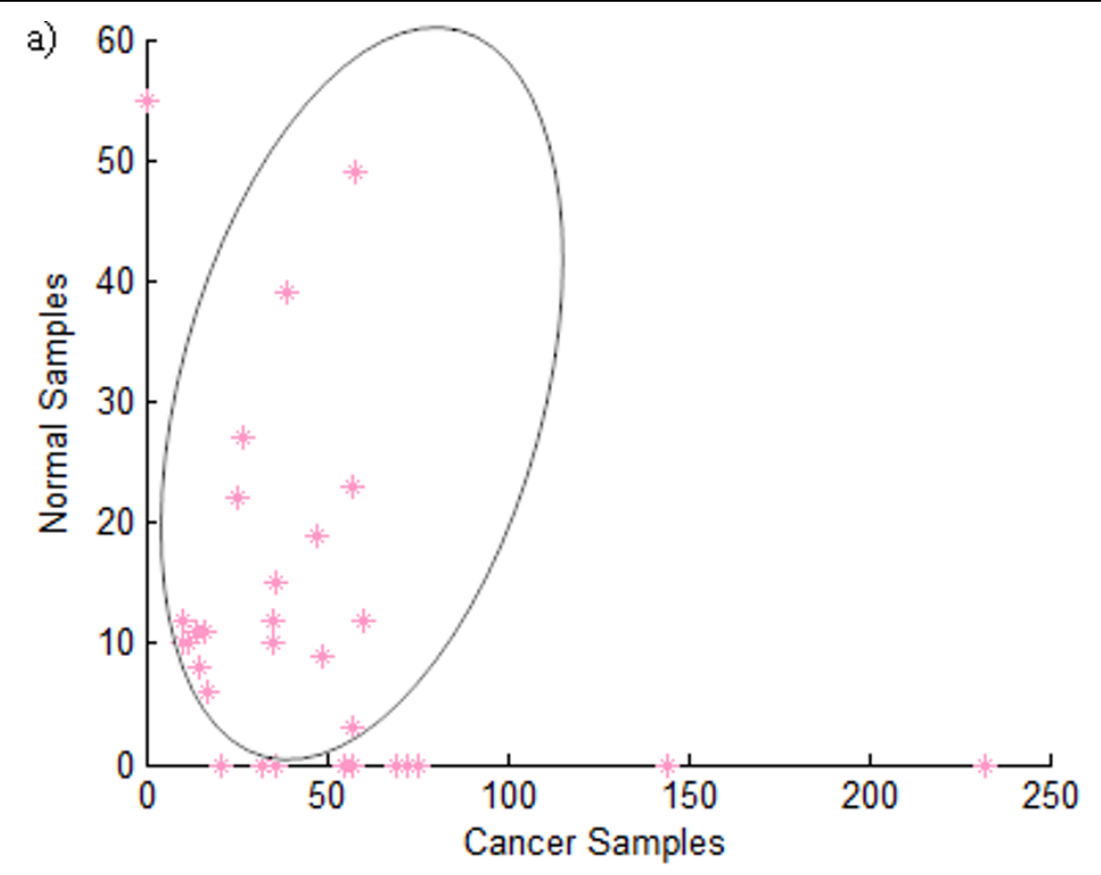

b)

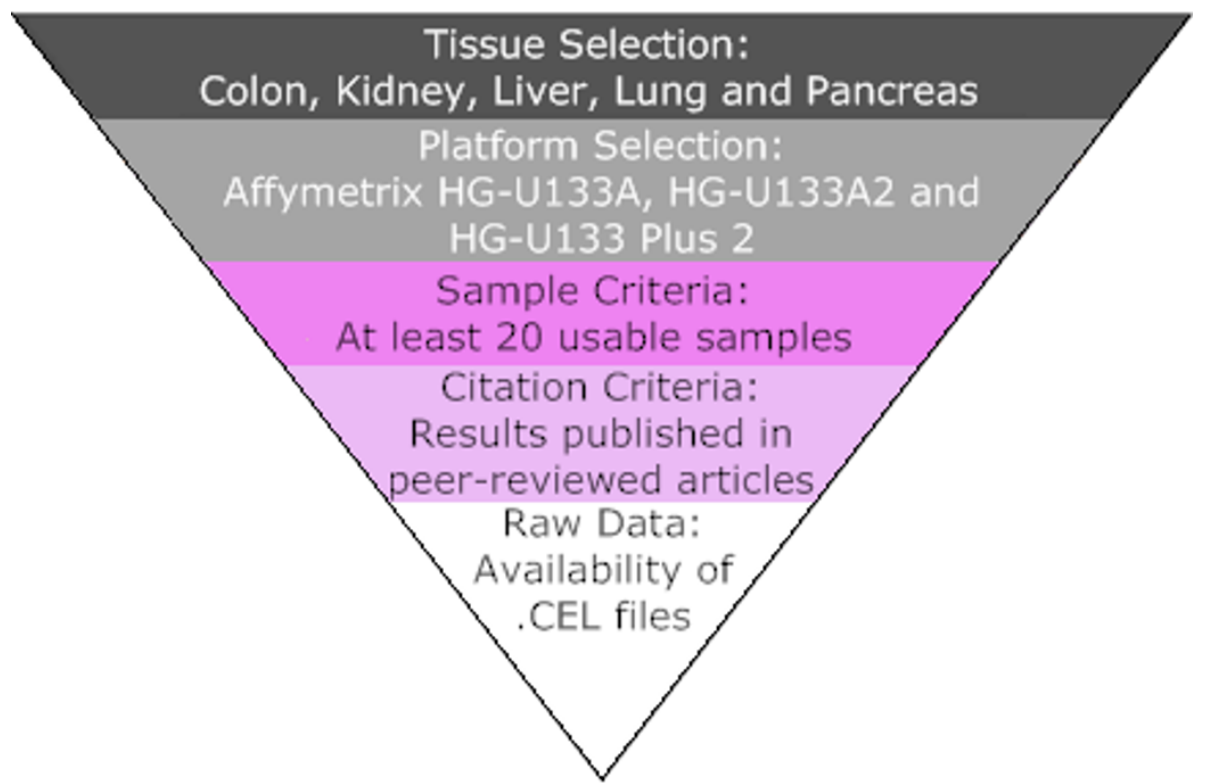

Figure 1 Overview of Microarray Datasets Used and Dataset Inclusion Criteria: a) Distribution of all Affymetrix microarray data used based on the number of cancer versus normal samples in each dataset. Datasets used for IV1/SAM1 test are shown inside the ellipse. Additional datasets included in SAM2 only are located on the axes. b) Selection method used for the inclusion of Affymetrix datasets used for the analyses in this study.

common to both kidney and colon cancers (butanoate and nitrogen metabolism). Complement and coagulation cascades were enriched in four out of the five tissues under study. These results show that both methods of integration are capable of reproducing a significant portion of the research literature on cellular pathways activated in cancer.

\section{Microarray results match cancer research literature with} low p-values

Next, we tested the SAM1, SAM2, IV1, and IV2 gene lists for PubMed hits associated with cancer. We conducted an automated PubMed abstract search for the genes in the aforementioned lists. All available abstracts in Pubmed were used excluding those that belonged to 
Table 2 The overlap among $n$ top-ranked genes between the IV1 and SAM1/SAM2 tests with corresponding p-values of the intersection, as well as among the top 400 genes between the similar approaches (IV1/IV2 and SAM1/SAM2)

\begin{tabular}{|c|c|c|c|c|c|c|c|c|c|c|c|c|}
\hline \multicolumn{13}{|c|}{ IV1 ก SAM1 } \\
\hline \multirow[t]{2}{*}{$\mathbf{n}$} & \multicolumn{2}{|l|}{ Colon } & \multicolumn{2}{|l|}{ Kidney } & \multicolumn{2}{|l|}{ Liver } & \multicolumn{2}{|l|}{ Lung } & \multicolumn{2}{|l|}{ Pancreas } & \multicolumn{2}{|l|}{ All } \\
\hline & Overlap & P-Value & Overlap & P-Value & Overlap & P-Value & Overlap & P-Value & Overlap & P-Value & Overlap & P-Value \\
\hline 10 & 3 & 1.01E-07 & 0 & 0.989487 & 5 & $9.98 \mathrm{E}-14$ & 2 & 4.49E-05 & 0 & 0.989487 & 0 & 0.989487 \\
\hline 50 & 11 & 8.67E-16 & 5 & $5.71 \mathrm{E}-06$ & 17 & $7.85 E-28$ & 14 & $1.44 \mathrm{E}-21$ & 8 & $1.49 \mathrm{E}-10$ & 6 & 2.05E-07 \\
\hline 100 & 26 & $4.68 \mathrm{E}-30$ & 23 & $3.04 \mathrm{E}-25$ & 24 & $8.09 \mathrm{E}-27$ & 34 & $4.21 \mathrm{E}-44$ & 17 & $1.93 \mathrm{E}-16$ & 18 & $7.94 \mathrm{E}-18$ \\
\hline 200 & 62 & $1.88 \mathrm{E}-57$ & 68 & $1.57 E-66$ & 53 & $9.78 \mathrm{E}-45$ & 93 & $2.56 \mathrm{E}-109$ & 34 & $8.96 \mathrm{E}-22$ & 64 & $2.00 \mathrm{E}-60$ \\
\hline 300 & 109 & $2.48 \mathrm{E}-91$ & 106 & $4.38 \mathrm{E}-87$ & 89 & $1.69 \mathrm{E}-64$ & 146 & $5.40 \mathrm{E}-150$ & 51 & $7.65 E-24$ & 103 & $6.46 E-83$ \\
\hline 400 & $132^{*}$ & 3.74E-98 & 146 & 1.41E-104 & 119 & $7.44 \mathrm{E}-72$ & 198 & $8.42 \mathrm{E}-181$ & 71 & $2.66 \mathrm{E}-26$ & 140 & $6.82 \mathrm{E}-97$ \\
\hline \multicolumn{13}{|c|}{ IV1 $\cap$ SAM2 } \\
\hline \multirow[t]{2}{*}{$\mathrm{n}$} & Colon & & Kidney & & Liver & & Lung & & Pancreas & & All & \\
\hline & Overlap & P-Value & Overlap & P-Value & Overlap & P-Value & Overlap & P-Value & Overlap & P-Value & Overlap & P-Value \\
\hline 10 & 3 & $1.01 \mathrm{E}-07$ & 0 & 0.989487 & 4 & $1.31 \mathrm{E}-10$ & 2 & 4.49E-05 & 0 & 0.989487 & 0 & 0.989487 \\
\hline 50 & 12 & 1.17E-17 & 5 & 5.71E-06 & 12 & $1.17 \mathrm{E}-17$ & 8 & 1.49E-10 & 8 & 1.49E-10 & 5 & 5.71E-06 \\
\hline 100 & 32 & $1.97 \mathrm{E}-40$ & 23 & $3.04 \mathrm{E}-25$ & 24 & $8.09 E-27$ & 28 & $2.09 E-33$ & 17 & $1.93 \mathrm{E}-16$ & 21 & $3.50 \mathrm{E}-22$ \\
\hline 200 & 67 & $5.51 \mathrm{E}-65$ & 66 & $1.88 \mathrm{E}-63$ & 43 & $5.97 E-32$ & 69 & $4.34 \mathrm{E}-68$ & 34 & $8.96 \mathrm{E}-22$ & 65 & $6.22 \mathrm{E}-62$ \\
\hline 300 & 111 & $3.32 \mathrm{E}-94$ & 116 & $1.54 \mathrm{E}-101$ & 60 & 4.00E-32 & 101 & $3.52 \mathrm{E}-80$ & 51 & $7.65 E-24$ & 101 & $3.52 \mathrm{E}-80$ \\
\hline 400 & $124^{*}$ & $9.02 \mathrm{E}-88$ & 168 & $1.02 \mathrm{E}-134$ & 86 & $1.19 \mathrm{E}-38$ & 149 & 1.67E-108 & 71 & $2.66 \mathrm{E}-26$ & 145 & $2.80 \mathrm{E}-103$ \\
\hline \multicolumn{13}{|c|}{ IV1 ก IV2 } \\
\hline \multirow[t]{2}{*}{$\mathrm{n}$} & Colon & & Kidney & & Liver & & Lung & & Pancreas & & All & \\
\hline & Overlap & P-Value & Overlap & P-Value & Overlap & P-Value & Overlap & P-Value & Overlap & P-Value & Overlap & P-Value \\
\hline 400 & $163^{*}$ & 1.39E-186 & 355 & 0 & No data & - & 144 & 3.97E-140 & No data & - & 280 & 0 \\
\hline \multicolumn{13}{|c|}{ SAM1 $\cap$ SAM2 } \\
\hline \multirow[t]{2}{*}{$\mathrm{n}$} & Colon & & Kidney & & Liver & & Lung & & Pancreas & & All & \\
\hline & Overlap & P-Value & Overlap & P-Value & Overlap & P-Value & Overlap & P-Value & Overlap & P-Value & Overlap & P-Value \\
\hline 400 & 176 & $1.92 \mathrm{E}-146$ & 284 & 0 & 253 & $6.86 \mathrm{E}-281$ & 241 & $3.15 E-257$ & No data & - & 262 & 2.34E-299 \\
\hline
\end{tabular}

* Only 338 genes are used for colon IV1

microarray-based research. Also excluded were abstracts that did not contain the word "cancer". A gene had to have at least one such PubMed abstract match to be considered as a literature search hit. The number of successful hits produced from the merged SAM methods and the IV tests intersected the research literature with significantly higher coverage than would be expected for randomly generated gene lists (Figure 5). The p-values shown in Figure 5 for the top 300 and 400 genes for all three methods were computed by using control gene lists obtained from the same Affymetrix platforms by randomly selecting lists of equal size $(300$ or 400) and averaging the number of hits over 100 iterations. The p-values for each tissue were then calculated using a normal distribution given the mean and standard deviation parameters of the randomly generated data. The p-value for the colon IV1 in the top 400 gene list was adjusted to a hundred iterations of 338 randomly chosen genes to account for the maximum available number of genes. The merged SAM methods produced gene lists that matched the research literature more accurately than the gene lists produced by the IV tests in four out of the five tissues under consideration. Additional File 1 contains the top 800 gene lists for the cancer types under consideration for SAM1 and IV1 approaches.

PubMed hits on gene lists presented by meta-analysis and merged SAM approaches fell inside and outside the intersections. Consider for example the case of colon cancer in IV1 and SAM1 gene lists. There were 93 hits on IV1 $\cap$ SAM1 ( $\mathrm{p}=1.19 \mathrm{E}-07), 103$ hits on IV1 - IV1 $\cap$ SAM1 $(\mathrm{p}=5.09 \mathrm{E}-02)$; and 205 hits on SAM1 - IV1 $\cap$ SAM1 ( $\mathrm{p}=2.32 \mathrm{E}-23)$.

As an additional control, the next top 400 genes (ranks 401-800) in each list, if available, were subjected to a similar PubMed abstract search. The p-values representing the results revealed decreased literature coverage of these genes compared to the first top 400 genes in all cases except for SAM2 results in lung tissue. In this test, majority of the IV results (except for lung 

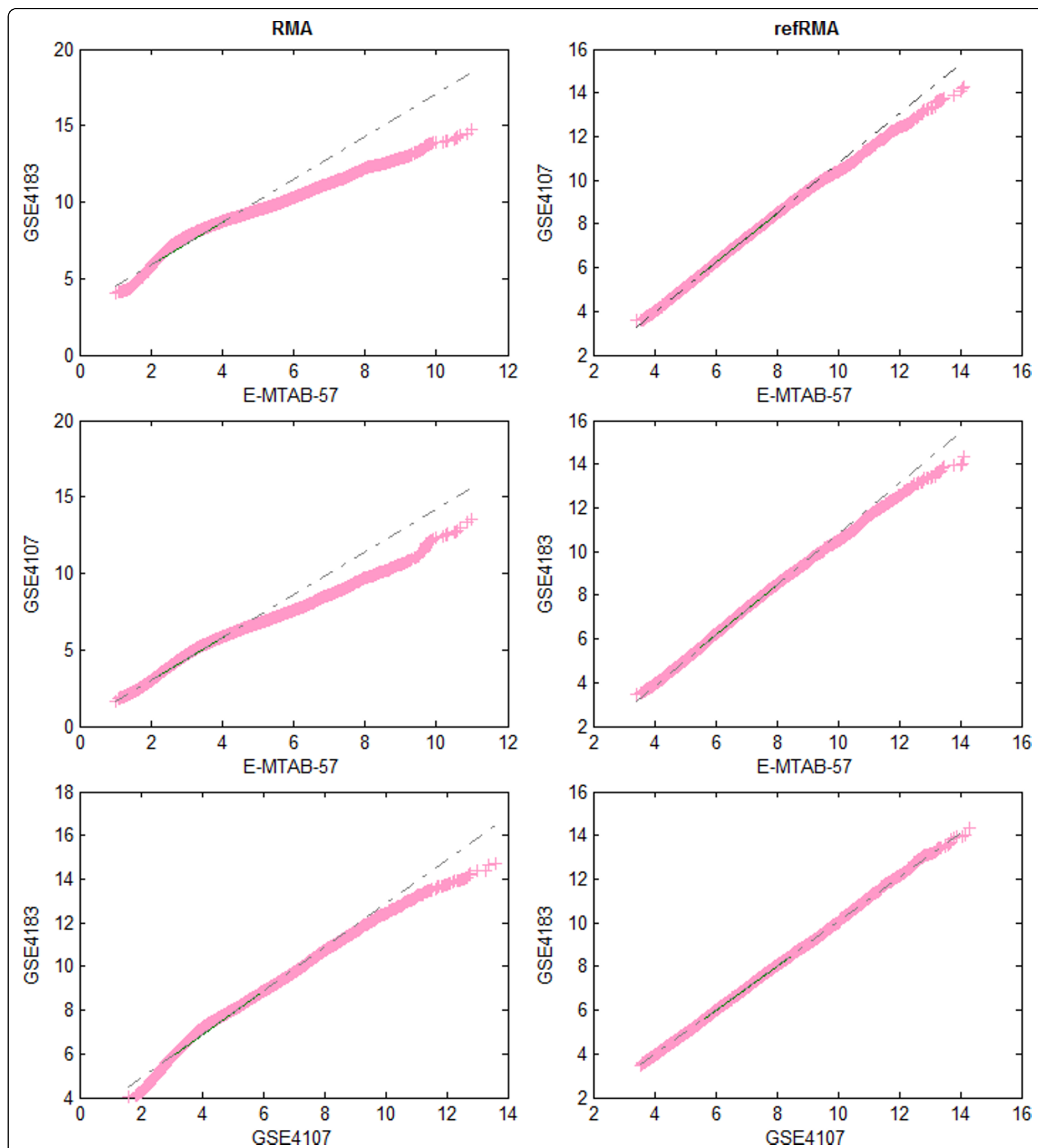

Figure 2 Quantile-Quantile Plots: Quantile-quantile plots indicating the distribution of three randomly chosen arrays from different colon datasets based on RMA (left) and refRMA (right) normalization.

and pancreas) dropped below the $0.0001 \mathrm{p}$-value threshold marked by a horizontal line in the figure.

Our results show that in the merged SAM approach, the more symmetric the datasets are in terms of containing both disease and control samples, the better is the match between gene lists produced by microarray analysis and the PubMed literature. Both SAM1 and SAM2 (containing asymmetric data) produced more significant $\mathrm{p}$-values per tissue than the average $\mathrm{p}$-value obtained from the SAM tests performed on the individual datasets for a given tissue (data not shown). The addition of single sample-type datasets (only cancer or only 
KEGG Pathways (Top 400 Genes)

Butanoate metabolism

Cell cycle

Citrate cycle (TCA cycle)

Complement and coagulation cascades

ECM-receptor interaction

Glycolysis / Gluconeogenesis

Nitrogen metabolism

p53 signaling pathway

PPAR signaling pathway

Purine metabolism

Pyrimidine metabolism

Synthesis and degradation of ketone bodies

Valine, leucine and isoleucine degradation
One carbon pool by folate

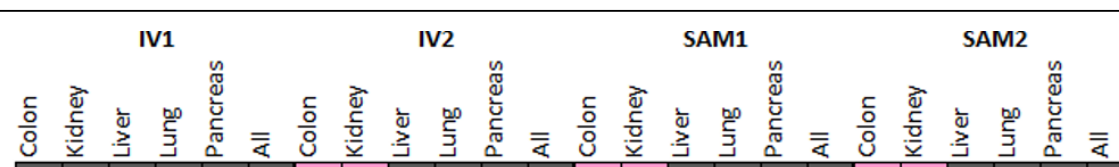

Figure 3 Enriched KEGG Pathways: A list of KEGG pathways, shown in pink, that appear to be statistically enriched according to the top 400 genes from IV1, IV2, SAM1 and SAM2 at a p-value cutoff of 0.05. Results are limited to pathways independently enriched in at least two of the tissues or in the combined test including all tissues.

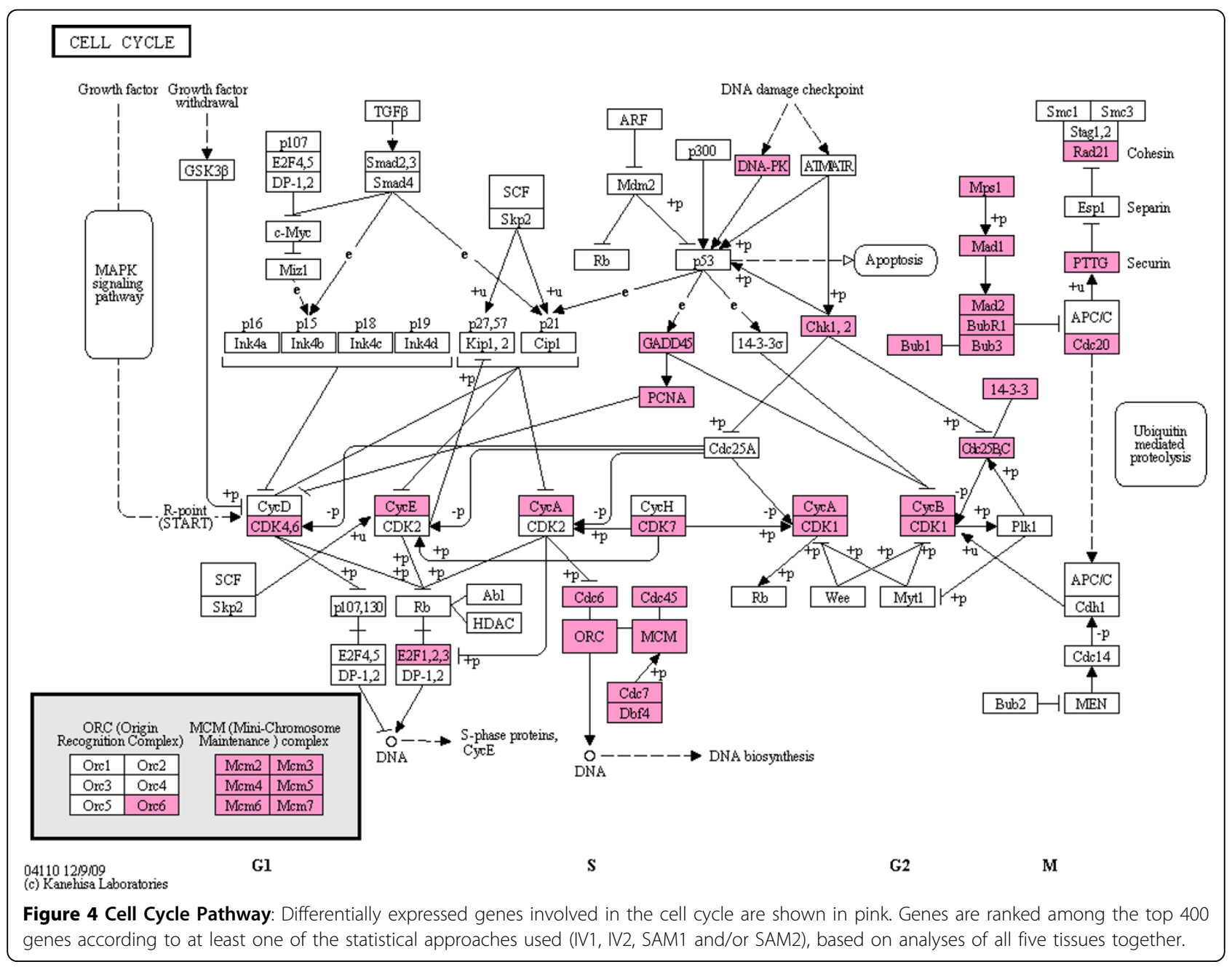




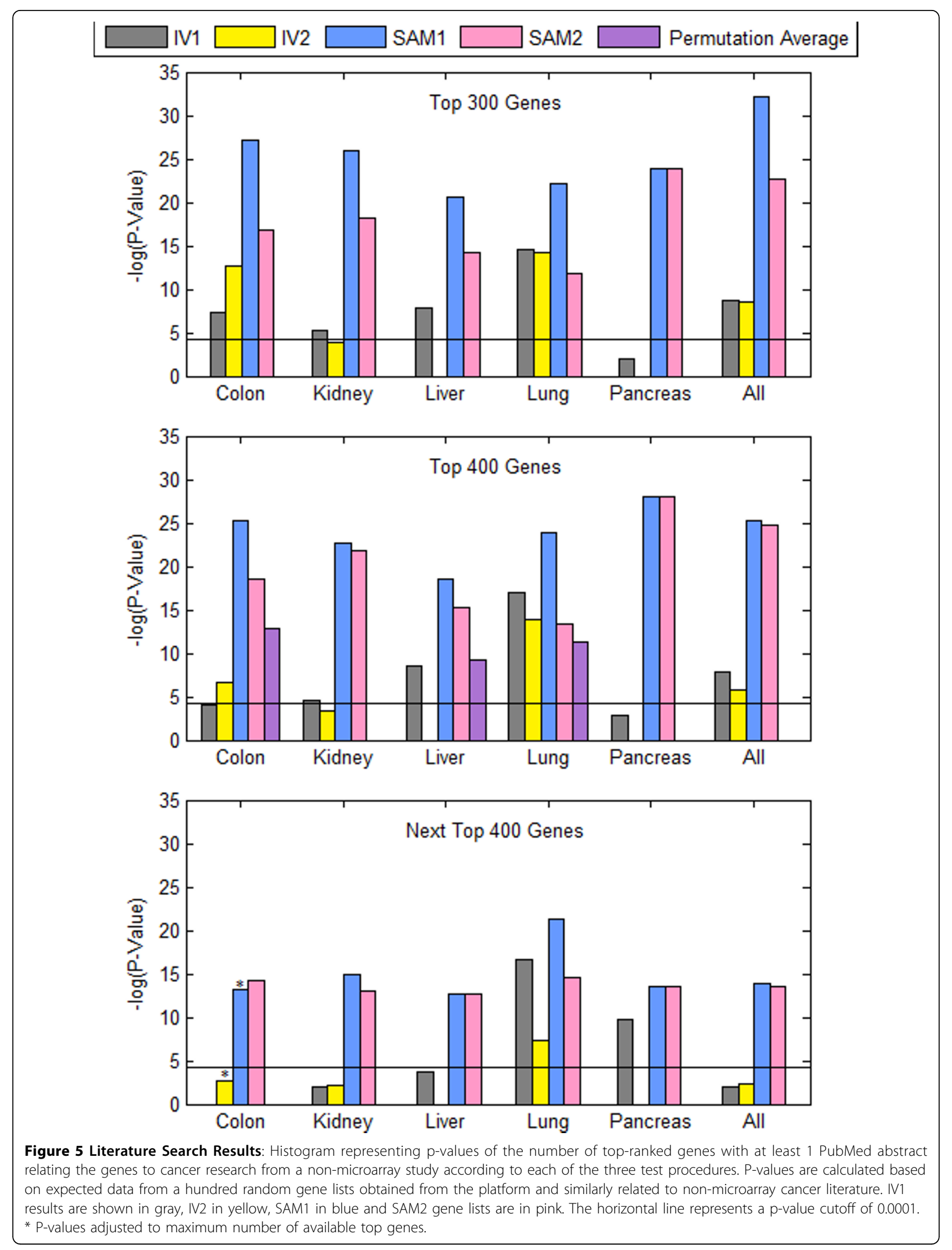


normal) in SAM2 resulted in fewer literature-associated gene lists than the SAM1 approach; however, the results improved when considering the top 400 genes as opposed to the top 300.

Next we considered an extreme case of SAM2, where the dataset was composed of disease samples from some studies and control samples from other studies. In this case, there would be no symmetric core in the dataset under consideration. To develop such purely asymmetric datasets, we deleted either the disease or the control samples of any symmetric study included in SAM2, considering all possible permutations for the datasets from three tissues: colon, liver and lung. The resulting gene lists were annotated with PubMed hits. We calculated an average number of PubMed hits over all possible combinations and corresponding $\mathrm{p}$-values. The results (shown in Figure 5 with purple bars) produced slightly fewer hits than the original SAM2 approach highlighting the importance of utilizing symmetric datasets when available as the core of the merged SAM technique. Nevertheless, even in this extreme case the probability for the match between literature and microarray gene lists to have occurred by random chance events was extremely small. It is clear from Figure 5 that the merged SAM analysis of purely asymmetric data results in prediction accuracy comparable to meta-analysis utilizing data with disease and control samples coming from the same labs.

\section{Discussion}

Meta-analysis approaches to microarray data aim to increase the statistical power of the results as well as increase reproducibility from individual studies [11]. Typical meta-analysis approaches combine results of independent datasets to produce a generalized outcome across these datasets. Meta-analysis approaches require both perturbed and control data within the same microarray datasets under consideration. However, the recent dramatic increase in public access microarray samples is mainly due to datasets containing no data on normal tissue. Noting that microarray samples on normal tissue are available in other public datastes, we wanted to explore the idea of picking samples from different datasets obtained with same/similar microarray chips and normalizing them together before the identification of significantly altered genes in normal to cancer comparison. The resulting merged SAM sacrifices the use of data from other platforms. However, it could be potentially useful for integrated analysis of cancer microarray datasets for which much of the available data is highly asymmetric. It is important to note that SAM analysis was chosen to determine the significant gene lists since it is believed to be superior to other microarray analysis methods.
A quick study of the GEO database clearly shows that microarray data for hormone-associated solid cancers such as breast, prostate and ovarian cancers are highly asymmetric. The more recent datasets increasingly come from studies for which one cancer subtype is compared to another cancer subtype and as a result contain no data from normal samples. We chose the five tissue types presented in this study because of the availability of data that could be used for both merged SAM and meta-analysis approaches. Previous studies have addressed the possible problems that arise from combining data across different technologies [36,37]. We have used the datasets obtained with similar chips to compare the performance of metaanalysis and merged the SAM approaches. The direct integration of data preceding the analysis as in the case of the merged SAM overcomes the problems associated with small sample sizes in individual studies. While data merging across similar chips sacrifices the inclusion of some of the genes not common to all platforms, it provides additional robustness since all samples are normalized together as opposed to being normalized separately per dataset [38].

We found that meta-analysis and merged SAM approaches yielded significant gene lists with intersecting common gene subsets that could not be plausibly obtained by chance. Both approaches matched automated PubMed abstract searches of research literature (excluding microarray studies) with very low p-values for random occurrence. However, the merged SAM approach replicated the existing literature much more accurately than the meta-analysis approach in five of the six cases under study. Addition of cDNA arrays into meta-analysis resulted in reduced overlap with the cancer literature. Meanwhile, the inclusion of asymmetric datasets also produced slightly less statistically significant results in merged SAM analyses, nevertheless, the approach still generated results that were at least as significant as the meta-analyses, again surpassing metaanalysis in five out of the six cases. Despite the addition of hundreds of samples from asymmetric sets, the merged SAM continued to perform well, matching literature as well as results of symmetric microarray data. We also showed that the match between microarray lists and the literature became less pronounced as lesser-ranked significant genes $(401-800)$ were used in the comparison. The gene lists obtained in all the tests were further validated by associating them with functional annotation through KEGG pathways. While individually each tissue possessed a unique list of pathways and processes with which it was associated, overall, cell division appeared to be the common driving factor to all tissues, as would be expected.

We used automated text searches as an instrument for validation of the prediction value of the two different 
approaches to integrate microarray data associated with cancer. Typical validation used in microarray analysis for illustrating relevance of gene list to disease state under consideration is usually via partitioning the dataset into learning, testing/validation subsets in a supervised learning approach [39-41]. However, it is relatively easy to differentiate between cancer and normal tissue with a variety of gene sets, but in many cases such sets are laboratory specific [42]. Research literature in cancer is rich with data on genes associated with this disease and the bulk of such data was collected by using research tools other than microarrays, and therefore, automated text search constituted an independent means of validating the microarray results. Approximately 520,000 PubMed abstracts were retrieved based on cancer association with genes from the relevant microarray platforms. Among those, 25,000 were associated with cancer but involved microarray studies and were therefore not included in our evaluation. The remaining PubMed hits were used to assign scores to the gene lists obtained and test the significance of these scores.

One reason for asymmetry in the current public access microarray data is that the goals of global gene expression quantification in cancer research shifted towards identifying significant genes associated with cancer subtypes [43-47]. The merged SAM analysis presented here is applicable to any microarray inquiry where there is a perturbed state (say cancer subtype 1 ) and control state (cancer subtype 2). We chose to illustrate the method of integration with cases where there was plenty of data for both meta-analysis and merged data approaches. Even when one aims to uncover differences in gene expression profile between two cancer subtypes, it is often useful to consider such differences between subtypes and control normal tissue samples [21]. Such triple comparisons reveal the original basis for the subtype differences that stem from normal to cancer transformations.

PubMed hits on gene lists produced by meta-analysis and merged SAM approaches fall on the intersections of such lists as well as outside the intersections, suggesting the use of both approaches whenever data is available. The top ranked 400 genes in both cases are highly statistically enriched with PubMed hits and for which the intersection between the two approaches had typically the lowest p-value. When considering the role of well studied genes such as hub genes or genes in public access cellular pathways, it is straightforward to project both gene lists onto known pathways to generate new hypotheses for experimental verification. The merged SAM technique provides a unique opportunity to obtain a candidate list for genes associated with a perturbed state in cases where the public microarray data is largely asymmetric.

\section{Conclusions}

Typical meta-analysis approaches allow for the use of various platforms at the expense of utilizing large amounts of data that exist in datasets containing either normal or cancer tissues only. Our merged SAM approaches have been shown to reproduce much of the known cancer literature while effectively being applied to asymmetrical microarray datasets. In our merged data approach, SAM analysis could be replaced by other widely used statistical methods, thus increasing the extent of the methodology. Such methods may include both parametric approaches such as PAGE [48] and T-profiler[49], or nonparametric approaches including GSEA [50] and rank products [51], among many others.. While many of the genes in our lists have already been associated with cancer, our approach sheds light on new genes which could play a pivotal role in cancer pathogenesis.

\section{Methods}

\section{Microarray dataset selection}

A total of 31 Affymetrix microarray datasets containing 1,768 unique samples from human cancer $(1,429)$ and corresponding healthy control tissues (339) were collected from the Gene Expression Omnibus (GEO; [2,3] and Array Express [4] online repositories (Additional File 2). Samples were selected for 5 different tissue types: colon, kidney, liver, lung and pancreas, then categorized into cancer and control subsets to allow for intra- and inter-tissue comparisons. The cancer samples were not restricted to a single type of malignancy in order to provide a generalized pathogenic approach shared by cancers. The microarray data were limited to those hybridized on the Affymetrix human microarray platforms HG-U133A, HG-U133A 2.0, and the HG-U133 Plus 2.0, due to the large overlap between the three platforms. In addition, the inclusion criteria restricted that each dataset was obtained from a peerreviewed study and contained a minimum of 20 usable microarray samples (Figure 1b).

\section{Normalization and differential expression}

For Affymetrix chips, raw microarray CEL files were read using the platform-compatible custom ENTREZG CDF file (version 12) [52] in order to obtain Entrez gene intensities. Where multiple replicates from the same source were available, the gene intensities were averaged across replicates. Nineteen out of 31 datasets contained samples for both the normal and cancer tissues and therefore could be used in meta-analysis. Individual datasets were background adjusted normalized with median polish using the robust multi-array analysis (RMA) in MATLAB [53]. For each tissue, the corresponding log-transformed 
data were transferred into $\mathrm{R}$ [54] and the metaGEM package [11] was utilized to conduct the meta-analysis using inverse variance (IV1). The IV model is based on a relative distance measurement computed as follows:

$$
\mathrm{d}(\mathrm{i})=\left[\mathrm{x}_{1}(\mathrm{i})-\mathrm{x}_{2}(\mathrm{i})\right] / \mathrm{s}(\mathrm{i})
$$

where $x_{1}(i)$ and $x_{2}(i)$ are the average levels of gene expression for gene (i) in states 1 and 2, respectively, and $\mathrm{s}(\mathrm{i})$ is the gene-specific pooled standard deviation which is equal to:

$$
\mathrm{s}(\mathrm{i})=\left[\left(\mathrm{n}_{1}-1\right) \mathrm{s}_{1}^{2}+\left(\mathrm{n}_{2}-1\right) \mathrm{s}_{2}^{2}\right] /\left(\mathrm{n}_{1}+\mathrm{n}_{2}-2\right)
$$

where $s_{1}$ and $s_{2}$ are the sample standard deviations of groups 1 and 2, while $\mathrm{n}_{1}$ and $\mathrm{n}_{1}$ are the number of samples in group 1 and 2, respectively. The false discovery rate (FDR) was set at $0.001 \%$. Moreover, the samr package [55] in $\mathrm{R}$ was used to conduct the significance analysis of microarrays (SAM) test [20] on each individual dataset. A hundred permutations were performed and results were restricted to significant genes with an FDR of 0 .

While IV analyzes each dataset separately before combining the results, SAM can be applied to previously merged data. This merger was achieved by using the refRMA algorithm [56], designed for large microarray datasets to compute the robust multichip averages. Similar to the classic RMA, background adjustment was applied to each sample from 909-array training set composed of all HG-U133 Plus 2.0 arrays used in this study. Quantile normalization was performed followed by median polishing. The outputs of this training process produces two archived vectors; a probe effect vector compiled from the individual log-scale probe affinity effects and a normalization vector compiled based on the transformed PM intensities. These vectors can then be extended to the samples from the other two platforms by using the predetermined group of arrays to estimate the effects and the average empirical distribution that should be used for the added data. The refRMA model is calculated as follows:

$$
\mathrm{T}\left(\mathrm{PM}_{\mathrm{ij}}\right)=\mathrm{e}_{\mathrm{i}}+\mathrm{a}_{\mathrm{j}}+\epsilon_{\mathrm{ij}} \text { such that } \mathrm{i}=1, \ldots, \mathrm{I} \text { (arrays) and } \mathrm{j}=1, \ldots, \mathrm{J} \text { (probes) }
$$

where $\mathrm{T}$ is the transformation for the background correction, normalization and $\log$ transformation of the perfect match intensities, $e_{i}$ is the $\log _{2}$ scale expression values of array $i, a_{j}$ is the $\log$ scale affinity effect of probe $\mathrm{j}$ and $\varepsilon_{\mathrm{ij}}$ is the error. A more detailed description can be found in [57].

The genes common to all three platforms are then chosen allowing for the integration of data from all three platforms together, limiting results to the 9,409 genes. To verify the application of refRMA to the added data compared to the original training set, one sample was randomly chosen from each of the three colon Affymetrix datasets that contained both healthy and cancer samples. Quantile-Quantile plots (Q-Q plots) were then generated for these arrays based on their individually normalized values (RMA) and collectively normalized values (refRMA). In each case, all gene expression values from one array are plotted against all gene expression values from the second array in order to assess the similarity in their distributions [14]. A merged SAM test was then applied to the combined data of each tissue using the same datasets included in the IV1 test based on the aforementioned parameters (100 permutations and 0 FDR).

As noted above, the IV test is limited to datasets that contain both cancer and normal tissues. The merged SAM method, however, allows for the inclusion of datasets containing solely normal or solely cancer samples. Thus, to test the effect of adding such datasets, microarray samples from all datasets of the same tissue were combined together and another series of SAM analyses were applied using the same test parameters as above. For the purpose of this paper, the first set of SAM tests, based on the data from the 19 datasets containing both normal and cancer tissues, is referred to as SAM1 (Figure 6). The second method in which all samples from the 31 datasets could be utilized is denoted as SAM2 (Figure 6). For each tissue, the lists of top 400 differentially expressed genes from the IV and both SAM tests were selected, based on the absolute relative distance measurement in gene expression. These gene lists were used to identify significantly enriched KEGG pathways at a p-value $\leq 0.05$ using DAVID Bioinformatics resources [32,33].

\section{Common transcriptional profiles across all five tissue types}

To identify consistent changes that are associated with multiple cancer tissue types, an IV1 test was conducted on all 19 Affymetrix datasets containing both cancer and normal samples together, regardless of tissue type. Similarly, a SAM test was performed on the same samples (SAM1) and another SAM test was applied to all 1,768 available Affymetrix samples from the 5 tissues considered (SAM2; Figure 6). The same test parameters were used as previously mentioned. After determining the genes that behave consistently across all the different cancer types, the top 400 genes were selected from the gene lists produced by each of the methods. Enriched KEGG pathways were identified for all lists at a p-value cutoff of 0.05 .

\section{Expanding IV analysis to cDNA data}

An additional 5 datasets using cDNA microarray platforms were obtained from GEO (Additional File 2). 


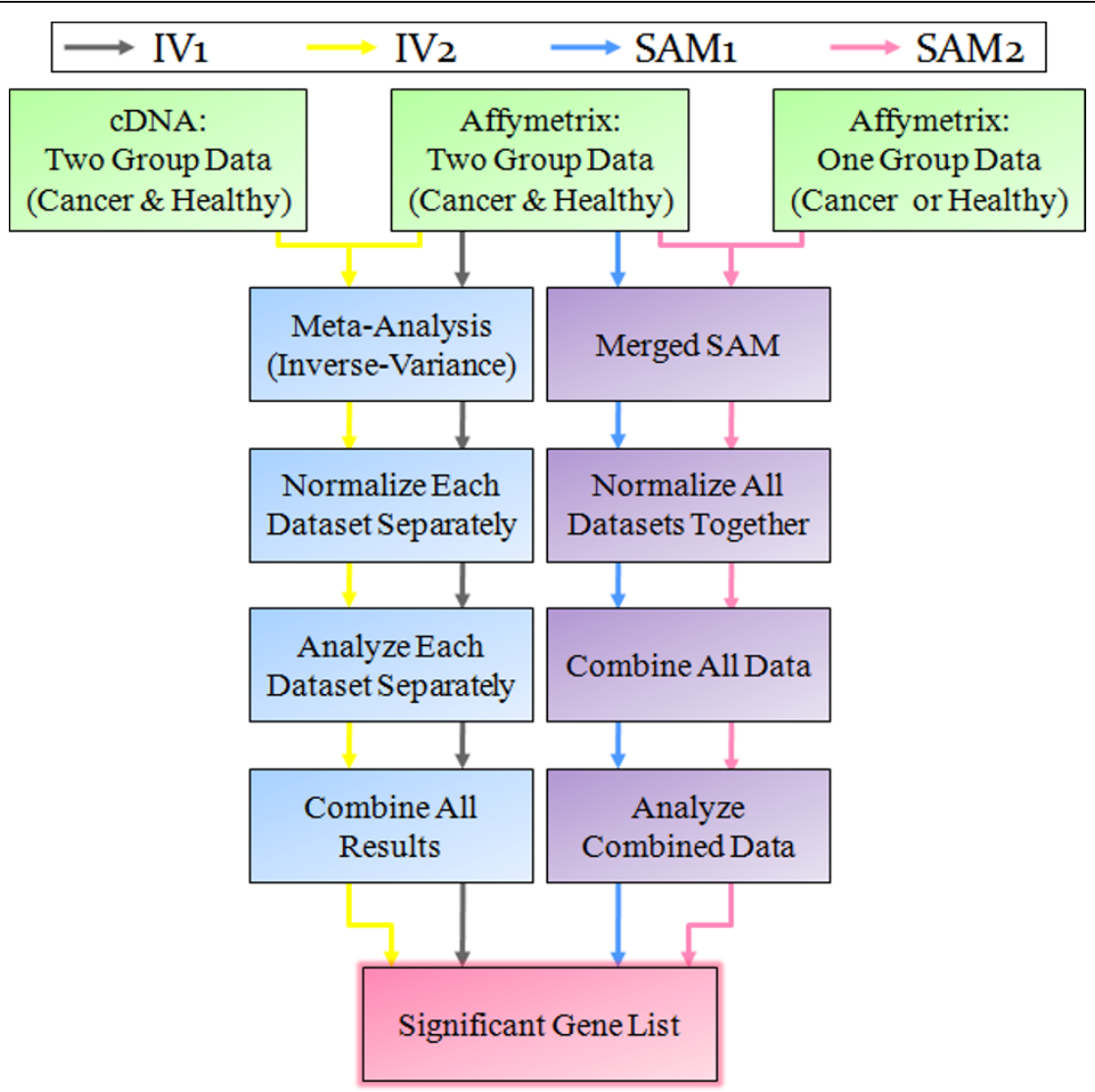

Figure 6 Workflow of the Analyses: Flowchart depicting the steps involved in each of the steps involved in each of the four analyses considered: IV1 (grey), IV2 (yellow), SAM1 (blue) and SAM2 (pink).

These datasets utilized different platforms and the conversion of data to Entrez IDs resulted in the study of varying number of genes per dataset as well as different total overlap with the common Affymetrix platform (shown in parentheses); GSE6988: 9,072 $(5,834)$ genes, GSE3: 12,452 (6,598) genes, GSE7367: 2118 $(1,301)$ genes, GSE2088: $13754(7,038)$ genes, and GSE8596: $6740(4,330)$ genes. The datasets contained cancer versus normal samples from colon, kidney and lung tissues for a total of 292 cancer and 169 normal samples. No publicly-accessible data could be found for the other two tissues. The IV analyses for these three tissues as well as the combined tissue test were re-run (IV2; Figure 6) to investigate the cost of excluding these datasets from the merged SAM approach that relies solely on Affymetrix data. Similar test parameters were applied, restricting results to genes with an FDR less than $0.001 \%$ and top 400 gene lists were utilized for identifying enriched KEGG pathways, as described above.

\section{Literature verification of results}

To determine the extent to which each method replicated the known cancer literature an automated text search was performed. A search of the gene symbol and the term "cancer NOT microarray" was conducted in PubMed abstracts for all genes available from the different platforms, limiting results to non-microarray literature. All gene lists obtained through IV and SAM analyses were then annotated with these results, identifying those genes that were cited in relation to cancer at least once from those that had no cancer association. A hundred random gene lists from the same platform of equal size to the lists under consideration were obtained and used as a control. The number of cancer-related genes in each of these random iteration was determined, and the mean and standard deviation were calculated from these values to obtain the parameters of a normal distribution. The expected value and the standard deviation were then used to compute the p-values for the significant association of each of our cancer gene lists with the known non-microarray literature. 


\section{Additional material}

Additional file 1: Top $\mathbf{8 0 0}$ Ranked Genes: Annotation of the top 800 genes for each tissue according to IV1 and SAM1analyses. Fold changes shown are based on overall values across all platforms and samples. Genes not shared by all three platforms are marked as unique.

Additional file 2: Microarray Samples Used in the Study: This file contains five worksheets that list all normal and cancer tissue microarray samples used including accession numbers of datasets, sample labels, sample tissue annotation and platform, in addition to malignancy description of cancer samples and available clinical annotation.

\section{List of abbreviations used}

IV: Inverse Variance; KEGG: Kyoto Encyclopedia of Genes and Genomes; SAM: significance analysis of microarrays.

\section{Competing interests}

The authors declare that they have no competing interests.

\section{Authors' contributions}

ND and AT conceptualized the research. ND implemented the algorithms and discussed results with AT and prepared the first draft of the manuscript. ND and AT both have read and approved the final manuscript.

\section{Availability}

The refRMA model is available by request from [56] and a MATLAB version is available by request from the corresponding author.

\section{Acknowledgements}

Authors thank Will Dampier, Erica Golemis, Andres Kriete, Lyle Ungar, Andrew Quong, and Ahmet Sacan for useful and insightful inputs. This research was supported by Calhoun fellowship to Noor Dawany as well as BioAdvance funds to Greater Philadelphia Bioinformatics Alliance.

Received: 15 March 2010 Accepted: 27 September 2010

Published: 27 September 2010

\section{References}

1. Zintzaras E, loannidis JP: Meta-analysis for ranked discovery datasets: theoretical framework and empirical demonstration for microarrays. Comput Biol Chem 2008, 32(1):38-46.

2. Barrett T, Troup DB, Wilhite SE, Ledoux P, Rudnev D, Evangelista C, Kim IF, Soboleva A, Tomashevsky M, Edgar R: NCBI GEO: mining tens of millions of expression profiles-database and tools update. Nucleic Acids Res 2007, 35 Database: D760-765.

3. Edgar R, Domrachev M, Lash AE: Gene Expression Omnibus: NCBI gene expression and hybridization array data repository. Nucleic Acids Res 2002, 30(1):207-210.

4. Brazma A, Parkinson $H$, Sarkans U, Shojatalab M, Vilo J, Abeygunawardena N, Holloway E, Kapushesky M, Kemmeren P, Lara GG, et al: ArrayExpress-a public repository for microarray gene expression data at the EBI. Nucleic Acids Res 2003, 31(1):68-71.

5. Rhodes DR, Barrette TR, Rubin MA, Ghosh D, Chinnaiyan AM: Meta-analysis of microarrays: interstudy validation of gene expression profiles reveals pathway dysregulation in prostate cancer. Cancer Res 2002, 62(15):4427-4433.

6. Rhodes DR, Yu J, Shanker K, Deshpande N, Varambally R, Ghosh D, Barrette T, Pandey A, Chinnaiyan AM: Large-scale meta-analysis of cancer microarray data identifies common transcriptional profiles of neoplastic transformation and progression. Proc Natl Acad Sci USA 2004, 101(25):9309-9314.

7. Smid M, Dorssers LC, Jenster G: Venn Mapping: clustering of heterologous microarray data based on the number of co-occurring differentially expressed genes. Bioinformatics 2003, 19(16):2065-2071.

8. Pihur V, Datta S: RankAggreg, an $\mathrm{R}$ package for weighted rank aggregation. BMC Bioinformatics 2009, 10:62.
9. DeConde RP, Hawley S, Falcon S, Clegg N, Knudsen B, Etzioni R: Combining results of microarray experiments: a rank aggregation approach. Stat Appl Genet Mol Biol 2006, 5:Article15.

10. Hong F, Breitling R, McEntee CW, Wittner BS, Nemhauser JL, Chory J: RankProd: a bioconductor package for detecting differentially expressed genes in meta-analysis. Bioinformatics 2006, 22(22):2825-2827.

11. Ramasamy A, Mondry A, Holmes CC, Altman DG: Key issues in conducting a meta-analysis of gene expression microarray datasets. PLoS Med 2008, 5(9):e184.

12. Choi JK, Yu U, Kim S, Yoo OJ: Combining multiple microarray studies and modeling interstudy variation. Bioinformatics 2003, 19(Suppl 1):i84-90.

13. Choi JK, Choi JY, Kim DG, Choi DW, Kim BY, Lee KH, Yeom Yl, Yoo HS, Yoo OJ, Kim S: Integrative analysis of multiple gene expression profiles applied to liver cancer study. FEBS Lett 2004, 565(1-3):93-100.

14. Warnat $P$, Eils $R$, Brors $B$ : Cross-platform analysis of cancer microarray data improves gene expression based classification of phenotypes. BMC Bioinformatics 2005, 6:265.

15. Hu PGC, Beyene J: Statistical methods for meta-analysis of microarray data: A comparative study. Inf Syst Front 2006, 8:9-20.

16. $\mathrm{Xu}$ L, Tan AC, Winslow RL, Geman D: Merging microarray data from separate breast cancer studies provides a robust prognostic test. BMC Bioinformatics 2008, 9:125.

17. Ertel A, Tozeren A: Human and mouse switch-like genes share common transcriptional regulatory mechanisms for bimodality. BMC Genomics 2008, 9:628.

18. Ertel A, Tozeren A: Switch-like genes populate cell communication pathways and are enriched for extracellular proteins. BMC Genomics 2008, 9:3.

19. Gormley M, Tozeren A: Expression profiles of switch-like genes accurately classify tissue and infectious disease phenotypes in model-based classification. BMC Bioinformatics 2008, 9:486.

20. Tusher VG, Tibshirani R, Chu G: Significance analysis of microarrays applied to the ionizing radiation response. Proc Natl Acad Sci USA 2001, 98(9):5116-5121.

21. Ertel A, Verghese A, Byers SW, Ochs M, Tozeren A: Pathway-specific differences between tumor cell lines and normal and tumor tissue cells. Mol Cancer 2006, 5(1):55

22. Sanga S, Broom BM, Cristini V, Edgerton ME: Gene expression metaanalysis supports existence of molecular apocrine breast cancer with a role for androgen receptor and implies interactions with ErbB family. BMC Med Genomics 2009, 2:59.

23. Gorlov IP, Byun J, Gorlova OY, Aparicio AM, Efstathiou E, Logothetis CJ: Candidate pathways and genes for prostate cancer: a meta-analysis of gene expression data. BMC Med Genomics 2009, 2:48.

24. Wirapati $P$, Sotiriou $C$, Kunkel S, Farmer P, Pradervand S, Haibe-Kains B, Desmedt C, Ignatiadis M, Sengstag T, Schutz F, et al: Meta-analysis of gene expression profiles in breast cancer: toward a unified understanding of breast cancer subtyping and prognosis signatures. Breast Cancer Res 2008, 10(4):R65.

25. $\mathrm{Xu} \mathrm{L}$, Geman D, Winslow RL: Large-scale integration of cancer microarray data identifies a robust common cancer signature. BMC Bioinformatics 2007, 8:275.

26. Hong Y, Ho KS, Eu KW, Cheah PY: A susceptibility gene set for early onset colorectal cancer that integrates diverse signaling pathways: implication for tumorigenesis. Clin Cancer Res 2007, 13(4):1107-1114.

27. Jones J, Otu H, Spentzos D, Kolia S, Inan M, Beecken WD, Fellbaum C, Gu X Joseph M, Pantuck AJ, et al: Gene signatures of progression and metastasis in renal cell cancer. Clin Cancer Res 2005, 11(16):5730-5739.

28. Su LJ, Chang CW, Wu YC, Chen KC, Lin CJ, Liang SC, Lin CH, Whang-Peng J, Hsu SL, Chen CH, et al: Selection of DDX5 as a novel internal control for Q-RT-PCR from microarray data using a block bootstrap re-sampling scheme. BMC Genomics 2007, 8:140.

29. Galamb O, Spisak S, Sipos F, Toth K, Solymosi N, Wichmann B, Krenacs T, Valcz G, Tulassay Z, Molnar B: Reversal of gene expression changes in the colorectal normal-adenoma pathway by NS398 selective COX2 inhibitor. Br J Cancer 2010, 102(4):765-773.

30. Yap YL, Lam DC, Luc G, Zhang XW, Hernandez D, Gras R, Wang E, Chiu SW, Chung LP, Lam WK, et al: Conserved transcription factor binding sites of cancer markers derived from primary lung adenocarcinoma microarrays. Nucleic Acids Res 2005, 33(1):409-421. 
31. Scotto L, Narayan G, Nandula SV, Arias-Pulido H, Subramaniyam S, Schneider A, Kaufmann AM, Wright JD, Pothuri B, Mansukhani M, et al: Identification of copy number gain and overexpressed genes on chromosome arm 20q by an integrative genomic approach in cervical cancer: potential role in progression. Genes Chromosomes Cancer 2008, 47(9):755-765.

32. Huang da W, Sherman BT, Lempicki RA: Systematic and integrative analysis of large gene lists using DAVID bioinformatics resources. Nat Protoc 2009, 4(1):44-57.

33. Dennis G Jr, Sherman BT, Hosack DA, Yang J, Gao W, Lane HC, Lempicki RA: DAVID: Database for Annotation, Visualization, and Integrated Discovery. Genome Biol 2003, 4(5):P3.

34. Kanehisa M, Goto S, Hattori M, Aoki-Kinoshita KF, Itoh M, Kawashima S, Katayama T, Araki M, Hirakawa M: From genomics to chemical genomics: new developments in KEGG. Nucleic Acids Res 2006, , 34 Database: D354-357.

35. Park MT, Lee SJ: Cell cycle and cancer. J Biochem Mol Biol 2003, 36(1):60-65

36. Jarvinen AK, Hautaniemi S, Edgren H, Auvinen P, Saarela J, Kallioniemi OP, Monni $O$ : Are data from different gene expression microarray platforms comparable? Genomics 2004, 83(6):1164-1168.

37. Kuo WP, Jenssen TK, Butte AJ, Ohno-Machado L, Kohane IS: Analysis of matched mRNA measurements from two different microarray technologies. Bioinformatics 2002, 18(3):405-412.

38. Nadon R, Shoemaker J: Statistical issues with microarrays: processing and analysis. Trends Genet 2002, 18(5):265-271.

39. Dyrskjot L, Kruhoffer M, Thykjaer T, Marcussen N, Jensen JL, Moller K, Orntoft TF: Gene expression in the urinary bladder: a common carcinoma in situ gene expression signature exists disregarding histopathological classification. Cancer Res 2004, 64(11):4040-4048.

40. Corvol JC, Pelletier D, Henry RG, Caillier SJ, Wang J, Pappas D, Casazza S, Okuda DT, Hauser SL, Oksenberg JR, et al: Abrogation of T cell quiescence characterizes patients at high risk for multiple sclerosis after the initial neurological event. Proc Natl Acad Sci USA 2008, 105(33):11839-11844.

41. Falt S, Merup M, Gahrton G, Lambert B, Wennborg A: Identification of progression markers in B-CLL by gene expression profiling. Exp Hematol 2005, 33(8):883-893.

42. Gormley M, Dampier W, Ertel A, Karacali B, Tozeren A: Prediction potential of candidate biomarker sets identified and validated on gene expression data from multiple datasets. BMC Bioinformatics 2007, 8:415.

43. Phillips HS, Kharbanda S, Chen R, Forrest WF, Soriano RH, Wu TD, Misra A, Nigro JM, Colman H, Soroceanu L, et al: Molecular subclasses of highgrade glioma predict prognosis, delineate a pattern of disease progression, and resemble stages in neurogenesis. Cancer Cell 2006, 9(3):157-173.

44. Groene J, Mansmann U, Meister R, Staub E, Roepcke S, Heinze M, Klaman I, Brummendorf T, Hermann K, Loddenkemper C, et al: Transcriptional census of 36 microdissected colorectal cancers yields a gene signature to distinguish UICC II and III. Int J Cancer 2006, 119(8):1829-1836.

45. Lin YH, Friederichs J, Black MA, Mages J, Rosenberg R, Guilford PJ, Phillips V, Thompson-Fawcett M, Kasabov N, Toro T, et al: Multiple gene expression classifiers from different array platforms predict poor prognosis of colorectal cancer. Clin Cancer Res 2007, $13(2$ Pt 1):498-507.

46. Turkheimer FE, Roncaroli F, Hennuy B, Herens C, Nguyen M, Martin D, Evrard A, Bours V, Boniver J, Deprez M: Chromosomal patterns of gene expression from microarray data: methodology, validation and clinical relevance in gliomas. BMC Bioinformatics 2006, 7:526.

47. Marty B, Maire V, Gravier E, Rigaill G, Vincent-Salomon A, Kappler M, Lebigot I, Djelti F, Tourdes A, Gestraud P, et al: Frequent PTEN genomic alterations and activated phosphatidylinositol 3-kinase pathway in basallike breast cancer cells. Breast Cancer Res 2008, 10(6):R101.

48. Kim SY, Volsky DJ: PAGE: parametric analysis of gene set enrichment. BMC Bioinformatics 2005, 6:144.

49. Boorsma A, Foat BC, Vis D, Klis F, Bussemaker HJ: T-profiler: scoring the activity of predefined groups of genes using gene expression data. Nucleic Acids Res 2005, , 33 Web Server: W592-595

50. Subramanian A, Tamayo P, Mootha VK, Mukherjee S, Ebert BL, Gillette MA, Paulovich A, Pomeroy SL, Golub TR, Lander ES, et al: Gene set enrichment analysis: a knowledge-based approach for interpreting genome-wide expression profiles. Proc Natl Acad Sci USA 2005, 102(43):15545-15550.
51. Breitling $R$, Armengaud $P$, Amtmann A, Herzyk P: Rank products: a simple, yet powerful, new method to detect differentially regulated genes in replicated microarray experiments. FEBS Lett 2004, 573(1-3):83-92.

52. Dai MH, Wang PL, Boyd AD, Kostov G, Athey B, Jones EG, Bunney WE, Myers RM, Speed TP, Akil H, et al: Evolving gene/transcript definitions significantly alter the interpretation of GeneChip data. Nucleic Acids Res 2005, 33(20).

53. Irizarry RA, Hobbs B, Collin F, Beazer-Barclay YD, Antonellis KJ, Scherf U, Speed TP: Exploration, normalization, and summaries of high density oligonucleotide array probe level data. Biostatistics 2003, 4(2):249-264.

54. R Development Core Team: R: A language and environment for statistical computing. R Foundation for Statistical Computing V, Austria. 2008 [http://www.R-project.org], ISBN 3-900051-07-0.

55. Tibshirani RCG, Hastie T, Narasimhan B: samr: SAM: Significance Analysis of Microarrays. R package version 1.26.[http://www-stat.stanford.edu/ tibs/ SAM].

56. Katz S, Irizarry RA, Lin X, Tripputi M, Porter MW: A summarization approach for Affymetrix GeneChip data using a reference training set from a large, biologically diverse database. BMC Bioinformatics 2006, 7:464.

57. Irizarry RA, Bolstad BM, Collin F, Cope LM, Hobbs B, Speed TP: Summaries of Affymetrix GeneChip probe level data. Nucleic Acids Res 2003, 31(4): e15.

doi:10.1186/1471-2105-11-483

Cite this article as: Dawany and Tozeren: Asymmetric microarray data produces gene lists highly predictive of research literature on multiple cancer types. BMC Bioinformatics 2010 11:483.

\section{Submit your next manuscript to BioMed Central and take full advantage of:}

- Convenient online submission

- Thorough peer review

- No space constraints or color figure charges

- Immediate publication on acceptance

- Inclusion in PubMed, CAS, Scopus and Google Scholar

- Research which is freely available for redistribution 